\title{
Factors Determining Affected Sites in Hidradenitis Suppurativa
}

\author{
Farida Benhadou $^{\mathrm{a}-\mathrm{c}}$ Axel Patrice Villani ${ }^{\mathrm{d}}$ e Philippe Guillem ${ }^{\mathrm{b}, c, f}$

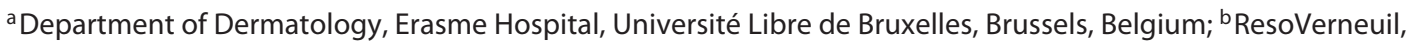

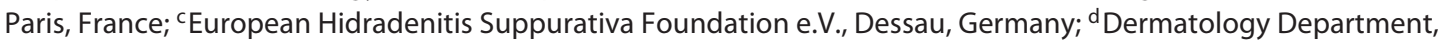 \\ Edouard Herriot Hospital, Hospices Civils de Lyon, Université Claude Bernard Lyon I, Lyon, France; ${ }^{\text {Groupe }}$ \\ HS-France de la Société Française de Dermatologie, Paris, France; ' Department of Surgery, Clinique du Val d'Ouest, \\ Lyon, France
}

Sir,

We have read the comments of Dr. van Straalen with great interest [1]. She raises 3 points of discussion:

The first point concerns gender as a confounding factor. We strongly agree that gender is a confounding factor. Initial univariate analysis of our data (Table 1) shows the sex differences observed. Gender is however not the only confounding factor and the same reasoning could be applied to many other confounders. Looking at the multivariate logistic regression analysis of the same data some differences appear while others disappear [2]. For instance, the association of female sex and HS of the groin became apparent, while male sex and axillary HS was not supported. Performing the analysis separately for men and women would have missed this.

The second point concerns the absence of a standard definition of anatomical sites involved in HS and subsequent inter-observer variation. Inter-observer variation was eliminated as all patients were assessed by the same physician [1]. Dr. van Straalen furthermore suggests combining sites in what she calls "more accepted" anatomical regions would be of interest [1]. The suggested regions are intended standardized photography [3]. We have explored this suggestion and redefined our cohort accordingly (Table 2). We find this new categorization interesting for the comparison of proportions with those of another cohort. It may however entail loss of information.

The third point raised concerns the logistic regression models. Dr. van Straalen relevantly points that Hurley stage is a categorical variable and therefore suggests to report odds for Hurley stage II and III with Hurley stage I as the reference category [1]. We would argue that Hurley staging is not only a categorical variable, but a semiquantitative ordered variable (stage III is more severe than stage II, which is itself more severe than stage I). When, therefore, the odds ratio for Hurley stages was quoted at 1.62 , it means that the considered risk increased by 1.62 from Hurley I to Hurley II and from Hurley II to Hurley III. 
In conclusion, we thank Dr. van Straalen for her critical reading of our work and agree with her that further standardized studies are clearly required.

\section{Key Message}

Further multivariate regression analyses and clearer definitions of anatomical sites are required to better associate affected sites and pathophysiological triggering factors.

\section{Conflict of Interest Statement}

P.G. received honoraria from AbbVie and Novartis as a consultant and provided lectures for AbbVie, Brothier, Cicaplus, Coloplast, Inresa and Novartis. A.P.V. received honoraria from Bailleul, GSK, Janssen and Novartis as a consultant. F.B. has no disclosure.

\section{Funding Sources}

The authors received no funding.

\section{Author Contributions}

F.B. and A.P.V.: critical revision of the work. P.G.: design of the work, acquisition, analysis and interpretation of the data, drafting of the work.

\section{References}

1 van Straalen KR. Conceivable Differences between the Sexes for the Associations of Patient Characteristics with Locations Affected by Hidradenitis Suppurativa. Dermatology. 2020 Apr;1-2.

2 Benhadou F, Villani AP, Guillem P. Which Factors Determine Affected Sites in Hidradenitis Suppurativa? Dermatology. 2020;236(1):15-20.

3 Zouboulis CC, Nogueira da Costa A. Standardized Photographic Documentation of Hidradenitis Suppurativa/Acne Inversa. Dermatology. 2019;235(1):51-4.
Table 1. Gender-specific characteristics in patients with hidradenitis suppurativa (univariate analysis; cohort described in [2])

\begin{tabular}{|c|c|c|c|}
\hline & $\begin{array}{l}\text { Males } \\
(n=411)\end{array}$ & $\begin{array}{l}\text { Females } \\
(n=727)\end{array}$ & $\begin{array}{l}\text { Signifi- } \\
\text { cance } \\
(p \\
\text { value })\end{array}$ \\
\hline \multicolumn{4}{|l|}{ Covariates } \\
\hline Age, years, mean \pm SD & $33 \pm 11$ & $31 \pm 10$ & 0.008 \\
\hline $\mathrm{BMI}, \mathrm{kg} / \mathrm{m}^{2}$, mean $\pm \mathrm{SD}$ & $27 \pm 5$ & $27 \pm 7$ & 0.329 \\
\hline Age at onset, years, mean \pm SD & $22 \pm 8$ & $20 \pm 8$ & $<0.001$ \\
\hline $\begin{array}{l}\text { Disease duration, years, mean } \pm \\
\text { SD }\end{array}$ & $11 \pm 9$ & $11 \pm 9$ & 0.652 \\
\hline Family history of HS, \% & 23.1 & 25.9 & 0.303 \\
\hline Smoking, \% & 88.6 & 74.3 & $<0.001$ \\
\hline \multicolumn{4}{|l|}{ Hurley stage, \% } \\
\hline I & 44.5 & 61.9 & \\
\hline II & 35.8 & 29.0 & $<0.001$ \\
\hline III & 19.7 & 9.1 & \\
\hline Inflammatory joint disease, $\%$ & 2.4 & 5.8 & 0.009 \\
\hline Inflammatory bowel disease, $\%$ & 0.7 & 2.9 & 0.015 \\
\hline Acne vulgaris, $\%$ & 21.9 & 16.2 & 0.018 \\
\hline Acne conglobate, $\%$ & 5.4 & 2.1 & 0.003 \\
\hline $\begin{array}{l}\text { Dissecting folliculitis of the scalp, } \\
\%\end{array}$ & 2.2 & 0.4 & 0.011 \\
\hline Epidermal cyst, \% & 23.6 & 14.4 & $<0.001$ \\
\hline \multicolumn{4}{|l|}{ Sites } \\
\hline Axilla, \% & 73.0 & 68.2 & 0.092 \\
\hline Neck, \% & 18.0 & 3.9 & $<0.001$ \\
\hline Retroauricular, \% & 14.6 & 7.1 & $<0.001$ \\
\hline Breast, \% & 7.1 & 28.6 & $<0.001$ \\
\hline Buttocks, \% & 32.1 & 30.4 & 0.547 \\
\hline Subgluteal, \% & 9.0 & 5.8 & 0.040 \\
\hline Intergluteal, $\%$ & 51.6 & 28.3 & $<0.001$ \\
\hline Groin, \% & 61.8 & 83.6 & $<0.001$ \\
\hline Inner thighs, $\%$ & 21.4 & 19.8 & 0.519 \\
\hline Pubis, \% & 20.2 & 23.9 & 0.147 \\
\hline Abdominal fold, $\%$ & 4.9 & 5.9 & 0.458 \\
\hline Genitals, \% & 19.2 & 22.3 & 0.225 \\
\hline Perineum, \% & 18.5 & 6.1 & $<0.001$ \\
\hline Anus, $\%$ & 17.8 & 10.7 & $<0.001$ \\
\hline Affected sites, $n$ (mean \pm SD) & $3.9 \pm 2.0$ & $3.6 \pm 1.8$ & 0.007 \\
\hline
\end{tabular}


Table 2. Multivariate analysis according to the site categorization

\begin{tabular}{|c|c|c|c|c|c|}
\hline \multicolumn{2}{|c|}{$\begin{array}{l}\text { Site categories according to } \\
\text { our original article }[1]\end{array}$} & \multicolumn{2}{|c|}{$\begin{array}{l}\text { Site categories according to } \\
\text { Zouboulis et al. [3] }\end{array}$} & \multirow{2}{*}{$\begin{array}{l}\text { Factors positively } \\
\text { associated with this site* } \\
\text { Pubis; Submammary } \\
\text { BMI; Hurley stage }\end{array}$} & \multirow{2}{*}{$\begin{array}{l}\text { Factors negatively } \\
\text { associated with this site* }\end{array}$} \\
\hline Armpits & $n=796(70.0 \%)$ & Armpits & $n=796(70.0 \%)$ & & \\
\hline $\begin{array}{l}\text { Groins } \\
\text { Inner thighs }\end{array}$ & $\begin{array}{l}n=862(75.7 \%) \\
n=232(20.4 \%)\end{array}$ & Groins & $n=919(80.8 \%)$ & $\begin{array}{l}\text { Woman; Age } \\
\text { Pubis; Genitals } \\
\text { Epidermal cyst } \\
\text { (BMI for inner thighs) }\end{array}$ & $\begin{array}{l}\text { Chest } \\
\text { Age at 1st occurrence } \\
\text { (BMI for groin) }\end{array}$ \\
\hline $\begin{array}{l}\text { Genitals } \\
\text { Perineum }\end{array}$ & $\begin{array}{l}n=241(21.2 \%) \\
n=120(10.5 \%)\end{array}$ & Genitals & $n=308(27.1 \%)$ & $\begin{array}{l}\text { Pubis; Groin; Anal area } \\
\text { Hurley } \\
\text { Age at 1st occurrence } \\
\text { (Woman for genitals) } \\
\text { (Smoking) } \\
\text { (DF of the scalp) }\end{array}$ & $\begin{array}{l}\text { Acne vulgaris } \\
\text { (abdominal fold) } \\
\text { (Woman for perineum) }\end{array}$ \\
\hline $\begin{array}{l}\text { Buttocks } \\
\text { Subgluteal fold }\end{array}$ & $\begin{array}{l}n=353(31.0 \%) \\
n=79(6.9 \%)\end{array}$ & Buttocks & $n=389(34.2 \%)$ & $\begin{array}{l}\text { Pubis; anal area } \\
\text { Age; (family history) } \\
\text { (Hurley and acne conglobata } \\
\text { for subgluteal) }\end{array}$ & $\begin{array}{l}\text { Armpit; BMI } \\
\text { Age at 1st occurrence }\end{array}$ \\
\hline $\begin{array}{l}\text { Pubis } \\
\text { Abdominal fold }\end{array}$ & $\begin{array}{l}n=257(22.6 \%) \\
n=53(4.7 \%)\end{array}$ & Pubis & $n=293(25.8 \%)$ & $\begin{array}{l}\text { Armpit; groin; buttocks } \\
\text { BMI } \\
\text { Genitals for pubis }\end{array}$ & $\begin{array}{l}\text { (Genitals for abdominal } \\
\text { fold) }\end{array}$ \\
\hline Breast & $n=237(20.8 \%)$ & $\begin{array}{l}\text { Chest } \\
\text { Submammary } \\
\text { (only in the } \\
727 \text { women) }\end{array}$ & $n=111(9.8 \%)$ & $\begin{array}{l}\text { Woman; BMI } \\
\text { Epidermal cyst } \\
\text { Acne conglobata } \\
\text { Groin, armpit, pubis } \\
\text { Buttocks, BMI }\end{array}$ & $\begin{array}{l}\text { Age } \\
\text { Groin }\end{array}$ \\
\hline
\end{tabular}

* Factors already found in our original article are shown in bold; factors described in our original article and not found to be significant with the revised categorization are indicated in brackets. 